\title{
Transmission of wireless backhaul signal in a cellular system with small moving cells
}

\author{
Hyoung-Keon Kim ${ }^{1}$, Yong-Suk Byun ${ }^{1}$, Geon-Woong Jung ${ }^{1}$ and Yong-Hwan Lee $2^{2^{*}}$ (D)
}

\begin{abstract}
Deployment of small moving cells (SMCs) has been considered in advanced cellular systems, where wireless backhaul links are required between base stations and SMCs. In this paper, we consider signal transmission by means of multiuser beamforming in the wireless backhaul link. We generate the beam weight in an eigen-direction of weighted combination of short- and long-term channel information of the backhaul link. The beam weight can maximize the average signal-to-leakage-plus-noise ratio (SLNR), while providing the transmission robust to SMC mobility. We analyze the performance of the proposed scheme in terms of the average signal-to-interference-plus-noise ratio (SINR) and optimize the transmit power by iterative water-filling. Finally, we verify the performance of the proposed scheme by computer simulation.
\end{abstract}

Keywords: MIMO, Moving cell, Backhaul

\section{Introduction}

Demands for mobile data traffic have rapidly been increasing with extensive deployment of multimedia services [1]. In particular, the growth rate of data traffic of users in mobility is more than two times that of total mobile data traffic [2]. Deployment of small moving cells (SMCs) has been proposed to provide desired quality of service (QoS) to users in mobility [3], where the base station (BS) of an SMC, referred to as S-BS, is installed in a vehicle and serves users in the vehicle. The S-BS can serve users without experiencing vehicular penetration loss (VPL), but it may require a wireless backhaul link for communication with a macro cell BS (M-BS).

The deployment of SMCs can enhance the system capacity by sharing transmission resource with macro cells [3-7]. It can increase the throughput of users near the cell edge [3]. The transmission resource can be partitioned in a non-orthogonal manner $[5,6]$, where the macro cell and SMCs can use the same resource for serving their users. Since the interference from the M-BS to SMC users can be somewhat reduced due to the effect of VPL, the overall spectral efficiency can be improved [5].

${ }^{*}$ Correspondence: ylee@snu.ac.k

${ }^{2}$ School of Electrical Engineering and INMC, Seoul National University, Gwanak-ro, Seoul, Republic of Korea

Full list of author information is available at the end of the article
However, most of previous studies did not consider the channel aging effect in the backhaul link. When an S-BS is in high mobility, the backhaul link capacity may significantly decrease mainly due to the channel mismatch problem [8]. The backhaul link can be a major bottleneck when it cannot provide the required capacity. Therefore, the channel aging can be a critical issue in the deployment of SMCs. Full-cache mode operation was considered for the deployment of SMCs [7], where an SMC can transmit cached data only. However, it may be applicable to a small portion of mobile traffic [9]. It was reported that the use of relay mode operation may not improve the transmission performance because of poor transmission performance in backhaul links [7].

It may be desirable to employ a transmission scheme to make the backhaul link robust to the channel aging effect. A two-stage multi-antenna beamforming scheme was proposed [10], where the outer beam weight is determined to suppress the interference among users, while the inner beam weight is determined to maximize the multiplexing gain. The outer beam weight can be determined by a set of eigenvectors of interference channel matrix corresponding to near-zero eigenvalues, while the inner beam weight can be determined to support zero-forcing (ZF) or singular value decomposition (SVD) transmission. However, this scheme is vulnerable to the channel aging since the beam weights are determined by using short-term 
channel state information (ST-CSI). Alternatively, the beam weight can be determined by using long-term (LT) or statistical CSI (e.g., spatial channel correlation matrix $(\mathrm{CCM}))[11,12]$. The beam weight can be determined by a set of eigenvectors of CCM corresponding to the largest eigenvalues [11]. The use of LT-CSI can provide the transmission quite efficient in high mobility environments, but not in low mobility environments [12]. The beam weight can be determined in a hybrid manner [13-15], where the outer and the inner beam weight are determined using CCM and ST-CSI, respectively. However, it may not be efficient for users in low or high mobility, compared to the use of ST-CSI or LT-CSI, respectively [13]. Temporal variation of backhaul channel can be estimated by using predictor antennas located in front of the backhaul link antennas of an SMC [16-18]. As the vehicle is moving forward, the location of the backhaul antenna passes through the location of the predictor antenna. This implies that the channel of the backhaul antennas and the predictor antennas may be correlated in spatial and temporal domain. The M-BS can predict the ST-CSI of backhaul antennas by exploiting the ST-CSI of predictor antennas [16]. However, the correlation between the channels of the predictor antennas and the backhaul antennas may noticeably vary in the presence of mobility [19]. When the correlation is not large, the channel prediction may not be effective for the estimation of ST-CSI of the backhaul channel.

In this paper, we consider signal transmission in the backhaul link of SMCs. We determine the beam weight by exploiting ST- and LT-CSI in consideration of SMC mobility. The M-BS estimates the signal-to-leakage-plusnoise ratio (SLNR) of the backhaul link and determines the beam weight to maximize the SLNR in an average sense. The beam weight makes the signal transmission in an eigen-direction of weighted combination of ST- and LTCSI. We analyze the average signal-to-interference-plus noise ratio (SINR) of the proposed scheme and use it to allocate the transmission power. Joint utilization of STand LT-CSI can provide the transmission performance robust to the variation of SMC mobility.

The remainder of this paper is organized as follows. The system model in consideration is described in Section 2. The proposed beamforming scheme is described in Section 3. The performance of the proposed scheme is verified by computer simulation in Section 4. Finally, conclusions are given in Section 5.

\section{System model}

Consider an orthogonal frequency division multiplexing (OFDM) cellular system that employs $K$ SMCs. We consider wireless backhaul links from an M-BS to $K$ S-BSs, where an M-BS and each of S-BSs employ $N_{T}$ and $N_{R}$ antennas, respectively, and $N_{T} \gg N_{R}$. Let $\mathbf{H}_{k} \in \mathbb{C}^{N_{R} \times N_{T}}$ be the backhaul channel from the M-BS to S-BS $k$ as illustrated in Fig. 1, and $\mathbf{R}_{k, r} \in \mathbb{C}^{N_{T} \times N_{T}}$ be the CCM corresponding to the $r$ th antenna of S-BS $k$ in spatial domain where $1 \leq k \leq K$. We assume that no correlation exists among the receive antennas (i.e., the CCM is an identity matrix) since the $\mathrm{S}-\mathrm{BS}$ may experience rich scattering compared to the M-BS. We also assume that S-BSs are sufficiently far away from the M-BS, yielding an identical CCM for different receive antennas (i.e., $\mathbf{R}_{k, r} \approx \mathbf{R}_{k}$ ). Then, it can be shown that

$$
\mathbf{H}_{k}=\tilde{\mathbf{H}}_{k} \mathbf{R}_{k}^{1 / 2}
$$

where $\tilde{\mathbf{H}}_{k}$ is an uncorrelated channel matrix whose elements are independent and identically distributed (i.i.d.) zero mean complex normal random variables with unit variance. Since the spatial correlation slowly changes in time and frequency domain [13], we can assume that $\mathbf{R}_{k}$ is unchanged during the time interval between the acquisition of ST-CSI and the data transmission.

Let $\hat{\mathbf{H}}_{k} \in \mathbb{C}^{N_{R} \times N_{T}}$ be the estimated ST-CSI by the M-BS, which has the same statistical characteristics as $\mathbf{H}_{k}$ in (1). We assume that the M-BS can perfectly estimate the STCSI, implying that the channel mismatch occurs only due to temporal variation of CSI. Then, $\mathbf{H}_{k}$ can be represented as [20]

$$
\mathbf{H}_{k}=\rho_{k} \hat{\mathbf{H}}_{k}+\sqrt{1-\rho_{k}^{2}} \mathbf{Z}_{k}
$$

where $\mathbf{Z}_{k} \in \mathbb{C}^{N_{R} \times N_{T}}$ is a temporal channel variation matrix which follows the same distribution as $\hat{\mathbf{H}}_{k}$ and $\rho_{k}$ is temporal correlation between $\hat{\mathbf{H}}_{k}$ and $\mathbf{H}_{k}$, represented as [20]

$$
\rho_{k}=J_{0}\left(\frac{2 \pi v_{k} f_{c} T_{k}}{c}\right) .
$$

Here $J_{0}$ is the 0th-order Bessel function of the first kind, $v_{k}$ is the velocity of S-BS $k, f_{c}$ is the carrier frequency, $T_{k}$ is the time interval between the ST-CSI estimation and the data transmission of S-BS $k$, and $c$ is the speed of light.

The signal received by S-BS $k$ can be represented as

$$
\mathbf{r}_{k}=\beta_{k} \mathbf{H}_{k} \mathbf{B}_{k} \mathbf{P}_{k} \mathbf{s}_{k}+\sum_{l \neq k} \beta_{k} \mathbf{H}_{k} \mathbf{B}_{l} \mathbf{P}_{l} \mathbf{s}_{l}+\mathbf{n}_{k}
$$

where $\beta_{k}$ is the large-scale fading coefficient, $\mathbf{B}_{k} \in \mathbb{C}^{N_{T} \times s_{k}}$ is the transmit beamforming matrix from the M-BS to S-BS $k, \mathbf{P}_{k} \in \mathbb{C}^{s_{k} \times s_{k}}$ is a diagonal matrix for power allocation of $\mathbf{B}_{k}, \mathbf{s}_{k} \in \mathbb{C}^{s_{k} \times 1}$ is the data vector, and $\mathbf{n}_{k} \in \mathbb{C}^{N_{R} \times 1}$ is an additive noise vector whose elements are i.i.d. zero mean complex normal random variables with variance $\sigma_{n}^{2}$. The S-BS $k$ can estimate $\mathbf{H}_{k} \mathbf{B}_{k} \mathbf{P}_{k}$ from demodulation reference signal (DM-RS) transmitted from the M-BS. It can decouple multiple streams of $\mathbf{s}_{k}$ by means of zero-forcing reception with a weight determined by

$$
\overline{\mathbf{W}}_{k}=\left(\beta_{k} \mathbf{H}_{k} \mathbf{B}_{k} \mathbf{P}_{k}\right)^{\dagger}
$$




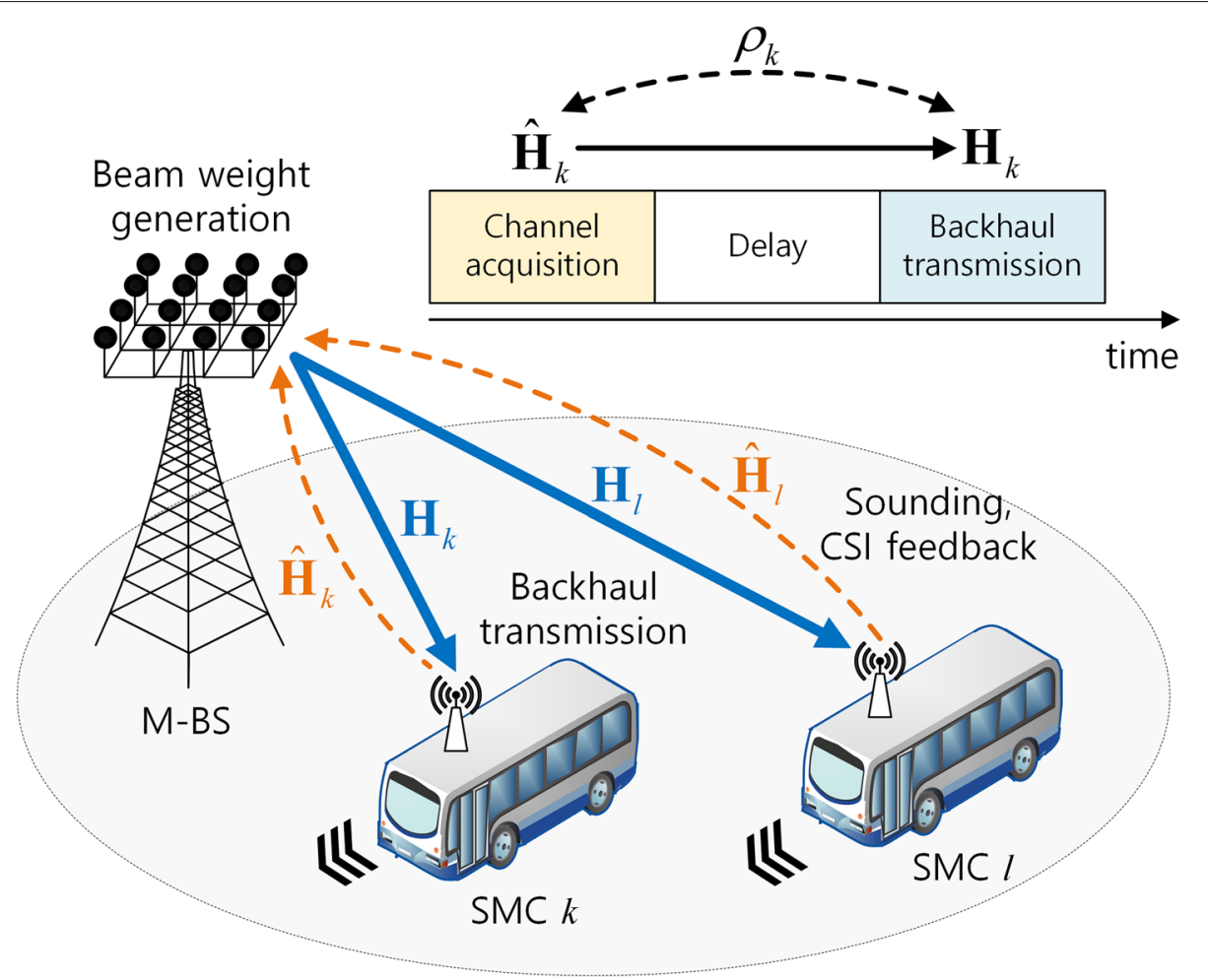

Fig. 1 A cellular system with SMCS

where the superscript $\dagger$ denotes the pseudo-inverse of a matrix. The corresponding received signal can be represented as

$$
\hat{\mathbf{x}}_{k}=\overline{\mathbf{w}}_{k} \mathbf{r}_{k} .
$$

\section{Proposed transmission scheme}

\subsection{Proposed beam design}

The SINR of the $i$ th stream received by S-BS $k$ can be represented as

$$
\gamma_{k, i}=\left(\left[E\left\{\overline{\mathbf{W}}_{k} \mathbf{T}_{k} \mathbf{T}_{k}^{H} \overline{\mathbf{W}}_{k}^{H}\right\}+E\left\{\overline{\mathbf{w}}_{k} \mathbf{n}_{k} \mathbf{n}_{k}^{H} \overline{\mathbf{W}}_{k}^{H}\right\}\right]_{i i}\right)^{-1}
$$

where $\mathbf{T}_{k} \equiv \sum_{l \neq k} \beta_{k} \mathbf{H}_{k} \mathbf{B}_{l} \mathbf{P}_{l} \mathbf{s}_{l},[\cdot]_{i i}$ denotes the $i$ th diagonal term of a matrix, and $(\cdot)^{H}$ denotes the Hermitian. It may not be easy to determine $\mathbf{B}_{k}$ maximizing the sum-rate $\sum_{k, i} \log _{2}\left(1+\gamma_{k, i}\right)$ since $\mathbf{B}_{k}$ and $\mathbf{B}_{l}$ should jointly be determined by (7). This problem can be alleviated by means of SLNR-based beamforming that minimizes leakage power instead of interference power $\mathbf{T}_{k}$ [21], where the leakage refers to the interference from the signal of a desired SMC to the other SMCs.

For ease of description, we assume that each signal transmission is equally powered, i.e., $\mathbf{P}_{k}=\sqrt{P_{k}} \mathbf{I}_{s_{k}}$, where
$\mathbf{I}_{s_{k}} \in \mathbb{C}^{s_{k} \times s_{k}}$ denotes an identity matrix. The desired signal power of the $i$ th stream received by S-BS $k$ can be represented as [22]

$$
\vartheta_{k, i}=\frac{\beta_{k}^{2} P_{k}}{\left[\left(\mathbf{B}_{k}^{H} \mathbf{H}_{k}^{H} \mathbf{H}_{k} \mathbf{B}_{k}\right)^{-1}\right]_{i i}} .
$$

The SLNR of S-BS $k$ can be represented as [21]

$$
\begin{aligned}
\varphi_{k}= & \frac{\sum_{i} \vartheta_{k, i}}{E\left\{N_{R} K \sigma_{n}^{2}+P_{k} \sum_{l, m \neq k} \beta_{l} \beta_{m} \mathbf{s}_{k}^{H} \mathbf{B}_{k}^{H} \mathbf{H}_{l}^{H} \mathbf{H}_{m} \mathbf{B}_{k} \mathbf{s}_{k}\right\}} \\
= & \frac{\sum_{i}\left[\left(\beta_{k}^{2} \mathbf{B}_{k}^{H} \mathbf{H}_{k}^{H} \mathbf{H}_{k} \mathbf{B}_{k}\right)^{-1}\right]_{i i}^{-1}}{\frac{N_{R} K \sigma_{n}^{2}}{P_{k}}+\operatorname{tr}\left(\mathbf{B}_{k}^{H} \sum_{j \neq k} \beta_{j}^{2} \mathbf{H}_{j}^{H} \mathbf{H}_{j} \mathbf{B}_{k}\right)} \\
= & \frac{\sum_{i}\left[\left(\beta_{k}^{2} \mathbf{B}_{k}^{H} \mathbf{H}_{k}^{H} \mathbf{H}_{k} \mathbf{B}_{k}\right)^{-1}\right]_{i i}^{-1}}{\operatorname{tr}\left(\mathbf{B}_{k}^{H}\left\{\frac{N_{R} K \sigma_{n}^{2}}{P_{k}} \mathbf{I}_{N_{T}}+\sum_{j \neq k} \beta_{j}^{2} \mathbf{H}_{j}^{H} \mathbf{H}_{j}\right\} \mathbf{B}_{k}\right)} \\
& \equiv \sum_{i} \frac{1}{\left[\mathbf{S}_{k}\right]_{i i}} \cdot \frac{1}{\operatorname{tr}\left(\mathbf{L}_{k}\right)}
\end{aligned}
$$


where $\operatorname{tr}(\cdot)$ denotes the trace of a matrix.

We consider temporal variation of the backhaul channel. The average of leakage $\mathbf{L}_{k}$ can be represented as

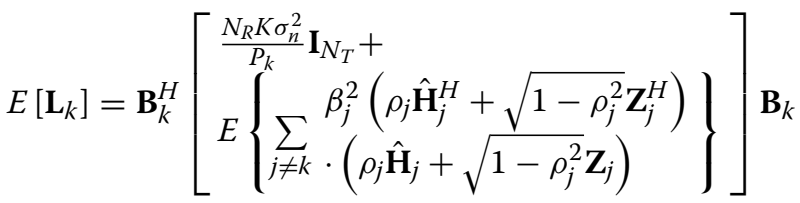

$$
\begin{aligned}
& =\mathbf{B}_{k}^{H}\left[\begin{array}{l}
\frac{N_{R} K \sigma_{n}^{2}}{P_{k}} \mathbf{I}_{N_{T}}+ \\
\sum_{j \neq k} \beta_{j}^{2}\left\{\rho_{j}^{2} \hat{\mathbf{H}}_{j}^{H} \hat{\mathbf{H}}_{j}+N_{R}\left(1-\rho_{j}^{2}\right) \mathbf{R}_{j}\right\}
\end{array}\right] \mathbf{B}_{k} \\
& =\mathbf{B}_{k}^{H}\left(\frac{N_{R} K \sigma_{n}^{2}}{P_{k}} \mathbf{I}_{N_{T}}+\mathbf{U}_{k} \mathbf{D}_{k} \mathbf{U}_{k}^{H}\right) \mathbf{B}_{k}
\end{aligned}
$$

where $\mathbf{U}_{k}$ and $\mathbf{D}_{k}$ are the eigenvectors and the eigenvalues of $\sum_{j \neq k} \beta_{j}^{2}\left\{\rho_{j}^{2} \hat{\mathbf{H}}_{j}^{H} \hat{\mathbf{H}}_{j}+N_{R}\left(1-\rho_{j}^{2}\right) \mathbf{R}_{j}\right\}$, respectively. The two terms in the parenthesis in the last equation of (10) can be removed by $\mathbf{B}_{k}$ when

$$
\begin{aligned}
\mathbf{B}_{k} & =\mathbf{U}_{k}\left(\frac{N_{R} K \sigma_{n}^{2}}{P_{k}} \mathbf{I}_{N_{T}}+\mathbf{D}_{k}\right)^{-1 / 2} \mathbf{B}_{k, 2} \\
& \equiv \mathbf{B}_{k, 1} \mathbf{B}_{k, 2}
\end{aligned}
$$

where $\mathbf{B}_{k, 2} \in \mathbb{C}^{N_{T} \times s_{k}}$. The corresponding leakage power can be represented as

$$
E\left\{\operatorname{tr}\left(\mathbf{L}_{k}\right)\right\}=\operatorname{tr}\left(\mathbf{B}_{k, 2}^{H} \mathbf{B}_{k, 2}\right) .
$$

The desired signal power in (9) can be represented as

$$
\begin{aligned}
& \sum_{i}\left[\mathbf{S}_{k}\right]_{i i}^{-1}=\sum_{i}\left[\left(\beta_{k}^{2} \mathbf{B}_{k, 2}^{H} \mathbf{B}_{k, 1}^{H} \mathbf{H}_{k}^{H} \mathbf{H}_{k} \mathbf{B}_{k, 1} \mathbf{B}_{k, 2}\right)^{-1}\right]_{i i}^{-1} \\
& =\sum_{i}\left[\left\{\begin{array}{l}
\beta_{k}^{2} \mathbf{B}_{k, 2}^{H}\left(\rho_{k} \mathbf{M}_{k}^{H}+\sqrt{1-\rho_{k}^{2}} \tilde{\mathbf{R}}_{k}^{1 / 2} \mathbf{Z}_{k}^{H}\right) \\
\cdot\left(\rho_{k} \mathbf{M}_{k}+\sqrt{1-\rho_{k}^{2}} \mathbf{Z}_{k} \tilde{\mathbf{R}}_{k}^{1 / 2}\right) \mathbf{B}_{k, 2}
\end{array}\right\}^{-1}\right]_{i i}^{-1}
\end{aligned}
$$

where $\tilde{\mathbf{R}}_{k}=\mathbf{B}_{k, 1}^{H} \mathbf{R}_{k} \mathbf{B}_{k, 1}$ and $\mathbf{M}_{k}=\hat{\mathbf{H}}_{k} \mathbf{B}_{k, 1} . \mathbf{B}_{k, 2}$ can be represented in an SVD form as

$$
\begin{aligned}
\mathbf{B}_{k, 2} & =\mathbf{U}_{B}\left[\begin{array}{ll}
\mathbf{D}_{B} & \mathbf{0}
\end{array}\right]^{T} \mathbf{V}_{B}^{H} \\
& \equiv \mathbf{U}_{B} \overline{\mathbf{D}}_{B} \mathbf{V}_{B}^{H}
\end{aligned}
$$

where $\mathbf{U}_{B} \in \mathbb{C}^{N_{T} \times N_{T}}$ and $\mathbf{V}_{B} \in \mathbb{C}^{s_{k} \times s_{k}}$ are the left and the right unitary matrix, respectively, $\mathbf{D}_{B} \in \mathbb{C}^{s_{k} \times s_{k}}$ is a diagonal matrix, $\mathbf{0}$ denotes a zero matrix, and the SMC index is omitted for simplicity of description. Then, (12) and (13) can be rewritten as, respectively,

$$
\sum_{i}\left[\mathbf{S}_{k}\right]_{i i}^{-1}=\sum_{i}\left[\mathbf{V}_{B}^{H} \overline{\mathbf{S}}_{k}^{-1} \mathbf{V}_{B}\right]_{i i}^{-1},
$$

$$
\begin{aligned}
E\left\{\operatorname{tr}\left(\mathbf{L}_{k}\right)\right\} & =\operatorname{tr}\left(\overline{\mathbf{D}}_{B}^{H} \overline{\mathbf{D}}_{B}\right) \\
& =\operatorname{tr}\left(\mathbf{D}_{B}^{2}\right)
\end{aligned}
$$

where $\overline{\mathbf{S}}_{k} \equiv \beta_{k}^{2} \overline{\mathbf{D}}_{B}^{H} \mathbf{U}_{B}^{H}\left(\rho_{k} \mathbf{M}_{k}^{H}+\sqrt{1-\rho_{k}^{2}} \tilde{\mathbf{R}}_{k}^{1 / 2} \mathbf{Z}_{k}^{H}\right)$. $\left(\rho_{k} \mathbf{M}_{k}+\sqrt{1-\rho_{k}^{2}} \mathbf{Z}_{k} \tilde{\mathbf{R}}_{k}^{1 / 2}\right) \mathbf{U}_{B} \overline{\mathbf{D}}_{B}$.

In the presence of unknown $\mathbf{Z}_{k}$, it may be desirable to determine $\mathbf{U}_{B}, \mathbf{V}_{B}$, and $\mathbf{D}_{B}$ to maximize the average SLNR, which can be represented as

$$
\begin{aligned}
\hat{\varphi}_{k}= & \frac{E\left\{\sum_{i}\left[\mathbf{S}_{k}\right]_{i i}^{-1}\right\}}{E\left\{\operatorname{tr}\left(\mathbf{L}_{k}\right)\right\}} \\
= & \frac{E\left\{\sum_{i}\left[\mathbf{V}_{B}^{H} \overline{\mathbf{S}}_{k}^{-1} \mathbf{V}_{B}\right]_{i i}^{-1}\right\}}{\operatorname{tr}\left(\mathbf{D}_{B}^{2}\right)}
\end{aligned}
$$

However, it may not be easy to calculate the mean of the numerator of (17) since $\overline{\mathbf{S}}_{k}^{-1}$ is the inverse of a non-central Wishart distributed matrix with $N_{R}$ degrees of freedom whose expectation may not exist [23].

We first consider a case when the M-BS has perfect information on $\mathbf{Z}_{k}$. Let $\mathbf{U}_{B}^{*}, \mathbf{V}_{B}^{*}$, and $\mathbf{D}_{B}^{*}$ be a solution of this case. Then, $\mathbf{V}_{B}^{*}$ can be determined by

$$
\mathbf{V}_{B}^{*}=\arg \max _{\mathbf{V}_{B}} \sum_{i}\left[\mathbf{V}_{B}^{H} \overline{\mathbf{S}}_{k}^{-1} \mathbf{V}_{B}\right]_{i i}^{-1} .
$$

Since $\overline{\mathbf{S}}_{k}$ is positive definite, it can be shown that $\sum_{i}\left[\mathbf{V}_{B}^{H} \overline{\mathbf{S}}_{k}^{-1} \mathbf{V}_{B}\right]_{i i}^{-1} \leq \operatorname{tr}\left(\mathbf{D}_{S}\right)$, where $\mathbf{D}_{S}$ is a diagonal matrix comprising eigenvalues of $\overline{\mathbf{S}}_{k}$ and the equality holds when $\mathbf{V}_{B}$ is an eigenvector matrix of $\overline{\mathbf{S}}_{k}$, denoted by $\mathbf{V}_{B}^{*}=\mathbf{U}_{S}$ (refer to the Appendix). The corresponding SLNR can be represented as

$$
\begin{aligned}
\varphi_{k} & =\frac{\operatorname{tr}\left(\mathbf{D}_{S}\right)}{\operatorname{tr}\left(\mathbf{D}_{B}^{2}\right)}=\frac{\operatorname{tr}\left(\overline{\mathbf{S}}_{k}\right)}{\operatorname{tr}\left(\mathbf{D}_{B}^{2}\right)} \\
& =\frac{\operatorname{tr}\left(\overline{\mathbf{D}}_{B}^{H} \mathbf{U}_{B}^{H}\left\{\beta_{k}^{2} \mathbf{B}_{k, 1}^{H} \mathbf{H}_{k}^{H} \mathbf{H}_{k} \mathbf{B}_{k, 1}\right\} \mathbf{U}_{B} \overline{\mathbf{D}}_{B}\right)}{\operatorname{tr}\left(\mathbf{D}_{B}^{2}\right)} .
\end{aligned}
$$

Let $\tilde{\mathbf{U}}_{k}$ and $\tilde{\mathbf{D}}_{k}$ be the matrix of eigenvectors and eigenvalues of $\beta_{k}^{2} \mathbf{B}_{k, 1}^{H} \mathbf{H}_{k}^{H} \mathbf{H}_{k} \mathbf{B}_{k, 1}$. With $\mathbf{U}_{B}=\tilde{\mathbf{U}}_{k} \tilde{\mathbf{U}}_{B}$, $\left\{\beta_{k}^{2} \mathbf{B}_{k, 1}^{H} \mathbf{H}_{k}^{H} \mathbf{H}_{k} \mathbf{B}_{k, 1}\right\}$ in (19) can be represented in a diagonal form. Then, (19) can be rewritten as

$$
\begin{aligned}
\varphi_{k} & =\frac{\operatorname{tr}\left(\overline{\mathbf{D}}_{B}^{H} \tilde{\mathbf{U}}_{B}^{H} \tilde{\mathbf{D}}_{k} \tilde{\mathbf{U}}_{B} \overline{\mathbf{D}}_{B}\right)}{\operatorname{tr}\left(\mathbf{D}_{B}^{2}\right)} \\
& \equiv \frac{\operatorname{tr}\left(\mathbf{B}_{k, 3}^{H} \tilde{\mathbf{D}}_{k} \mathbf{B}_{k, 3}\right)}{\operatorname{tr}\left(\mathbf{B}_{k, 3}^{H} \mathbf{B}_{k, 3}\right)} .
\end{aligned}
$$


Since $\mathbf{B}_{k, 3}=\left[\tilde{\mathbf{U}}_{B} \mathbf{D}_{B} \mathbf{0}\right]^{H}=\left[\begin{array}{ll}\mathbf{I}_{s_{k}} & \mathbf{0}\end{array}\right]^{H}$ maximizes $\varphi_{k}$ [21], we can determine $\mathbf{U}_{B}^{*}=\tilde{\mathbf{U}}_{k} \tilde{\mathbf{U}}_{B}=\tilde{\mathbf{U}}_{k}$ and $\mathbf{D}_{B}^{*}=\mathbf{I}_{s_{k}}$.

Consider a case when $\mathbf{Z}_{k}$ is unknown to the M-BS. Although the M-BS cannot jointly determine $\mathbf{U}_{B}^{*}$ and $\mathbf{V}_{B}^{*}$, it can determine $\mathbf{U}_{B}^{*}$ from the SLNR in an average sense if $\mathbf{V}_{B}^{*}$ is known (and vice versa). Assuming that $\mathbf{V}_{B}^{*}$ is known to the M-BS, the average SLNR can be represented as

$$
\begin{aligned}
& \hat{\varphi}_{k}=\frac{E\left\{\operatorname{tr}\left(\overline{\mathbf{S}}_{k}\right)\right\}}{\operatorname{tr}\left(\overline{\mathbf{D}}_{B}^{2}\right)} \\
& =\frac{\operatorname{tr}\left(\mathbf{D}_{B}^{H} \mathbf{U}_{B}^{H} \beta_{k}^{2}\left\{\begin{array}{l}
\rho_{k}^{2} \mathbf{M}_{k}^{H} \mathbf{M}_{k}+ \\
N_{R}\left(1-\rho_{k}^{2}\right)
\end{array} \tilde{\mathbf{R}}_{k}\right\} \mathbf{U}_{B} \mathbf{D}_{B}\right)}{\operatorname{tr}\left(\overline{\mathbf{D}}_{B}^{2}\right)}
\end{aligned}
$$

Using the same approach as in the case when $\mathbf{Z}_{k}$ is known to the M-BS, it can be shown that the average SLNR can be maximized with $\hat{\mathbf{U}}_{B}^{*}=\overline{\mathbf{U}}_{k}$ and $\hat{\mathbf{D}}_{B}^{*}=$ $\mathbf{I}_{s_{k}}$, where $\overline{\mathbf{U}}_{k}$ is an eigenvector matrix of $\rho_{k}^{2} \mathbf{M}_{k}^{H} \mathbf{M}_{k}+$ $N_{R}\left(1-\rho_{k}^{2}\right) \tilde{\mathbf{R}}_{k}$. On the other hand, when $\mathbf{U}_{B}^{*}$ is known to the M-BS, $\overline{\mathbf{S}}_{k}^{-1}$ becomes a diagonal matrix. Then, it can be shown that $\hat{\mathbf{V}}_{B}^{*}=\mathbf{I}_{s_{k}}$ is a solution of (18). The beam weight can be determined by

$$
\begin{aligned}
& \mathbf{B}_{k}=\mathbf{B}_{k, 1} \mathbf{B}_{k, 2} \\
& =\mathbf{B}_{k, 1} \hat{\mathbf{U}}_{B}^{*}\left[\hat{\mathbf{D}}_{B}^{*} \mathbf{0}\right]^{T} \hat{\mathbf{V}}_{B}^{*} \\
& =\mathbf{U}_{k}\left(\frac{N_{R} K \sigma_{n}^{2}}{P_{k}} \mathbf{I}_{N_{T}}+\mathbf{D}_{k}\right)^{-1 / 2}\left[\overline{\mathbf{U}}_{k}\right]_{: 1: s_{k}} \text {. }
\end{aligned}
$$

Instead of the proposed approach, a sub-optimal solution of (18) can be obtained by searching a unitary matrix that diagonalizes $\overline{\mathbf{S}}_{k}^{-1}$ in an average sense when $\mathbf{Z}_{k}$ is unknown. The solution can be obtained by means of approximated joint diagonalization (AJD) that searches for a unitary matrix by jointly diagonalizing a large number of samples of $\overline{\mathbf{S}}_{k}^{-1}$ (or $\overline{\mathbf{S}}_{k}$ ) in a Monte-Carlo manner [24]. However, the computational complexity may become extremely large as the number of samples increases.

\subsection{Performance analysis}

We determine the transmit power based on the SINR by means of water-filling. We may separate the power of desired signal from the interference plus noise term in (7). The SINR can be represented as

$$
\gamma_{k, i}=\frac{\left[\mathbf{P}_{k}^{2}\right]_{i i}}{\left[E\left\{\mathbf{W}_{k} \mathbf{T}_{k} \mathbf{T}_{k}^{H} \mathbf{W}_{k}^{H}\right\}+E\left\{\mathbf{W}_{k} \mathbf{n}_{k} \mathbf{n}_{k}^{H} \mathbf{W}_{k}^{H}\right\}\right]_{i i}}
$$

where $\mathbf{W}_{k}=\beta_{k}^{-1}\left(\mathbf{B}_{k}^{H} \mathbf{H}_{k}^{H} \mathbf{H}_{k} \mathbf{B}_{k}\right)^{-1} \mathbf{B}_{k}^{H} \mathbf{H}_{k}^{H}$. The interference power term in the denominator of (23) can be represented as

$$
\begin{aligned}
& \mathbf{W}_{k} \mathbf{T}_{k} \mathbf{T}_{k}^{H} \mathbf{W}_{k}^{H} \\
& =\mathbf{W}_{k} \sum_{l, m \neq k} \beta_{k}^{2}\left(\mathbf{H}_{k} \mathbf{B}_{l} \mathbf{P}_{l} \mathbf{s}_{l} \mathbf{s}_{m}^{H} \mathbf{P}_{m}^{H} \mathbf{B}_{m}^{H} \mathbf{H}_{k}^{H}\right) \mathbf{W}_{k}^{H} \\
& =\mathbf{W}_{k} \sum_{l, m \neq k} \beta_{k}^{2}\left\{\begin{array}{c}
\left(\rho_{k} \hat{\mathbf{H}}_{k}+\sqrt{1-\rho_{k}^{2}} \mathbf{Z}_{k}\right) \mathbf{B}_{l} \mathbf{P}_{l} \mathbf{s}_{l} \cdot \\
\mathbf{s}_{m}^{H} \mathbf{P}_{m}^{H} \mathbf{B}_{m}^{H}\left(\rho_{k} \hat{\mathbf{H}}_{k}^{H}+\sqrt{1-\rho_{k}^{2}} \mathbf{Z}_{k}^{H}\right)
\end{array}\right\} \mathbf{W}_{k}^{H} .
\end{aligned}
$$

Since $\mathbf{W}_{k}$ and $\mathbf{H}_{k} \mathbf{B}_{l}$ are correlated to each other, it may be desirable to split $\mathbf{H}_{k} \mathbf{B}_{l}$ into two terms: one term that includes $\mathbf{W}_{k}^{\dagger}$ and the other term that is independent of $\mathbf{W}_{k}$. The term $\mathbf{Z}_{k} \mathbf{B}_{l} \mathbf{P}_{l}$ can be represented as

$$
\mathbf{Z}_{k} \mathbf{B}_{l} \mathbf{P}_{l} \stackrel{d}{=} \mathbf{Z}_{k} \mathbf{B}_{k} \Psi_{l}+\mathbf{X}_{l}
$$

where $\Psi_{l}$ is a constant matrix and $\mathbf{X}_{l}$ is a matrix comprising independent normal random row vectors, while satisfying

$$
\left\{\begin{aligned}
\Psi_{l}=\left(\mathbf{B}_{k}^{H} \mathbf{R}_{k} \mathbf{B}_{k}\right)^{-1} \mathbf{B}_{k}^{H} \mathbf{R}_{k} \overline{\mathbf{B}}_{l} \\
E\left\{\mathbf{X}_{l}\right\}=\mathbf{0} \\
\begin{array}{rl}
E\left\{\mathbf{X}_{l}^{H} \mathbf{X}_{l} / N_{R}\right\} & =\overline{\mathbf{B}}_{l}^{H} \mathbf{R}_{k} \overline{\mathbf{B}}_{l}-\Psi_{l}^{H} \mathbf{B}_{k}^{H} \mathbf{R}_{k} \mathbf{B}_{k} \Psi_{l} \\
& \equiv \mathbf{Y}_{l}
\end{array} .
\end{aligned}\right.
$$

Here, $\overline{\mathbf{B}}_{l} \equiv \mathbf{B}_{l} \mathbf{P}_{l}$. Then, it can be shown that

$$
\begin{aligned}
& E\left\{\mathbf{W}_{k} \mathbf{T}_{k} \mathbf{T}_{k}^{H} \mathbf{W}_{k}^{H}\right\}
\end{aligned}
$$

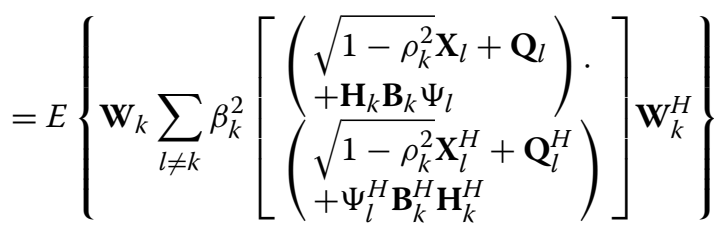

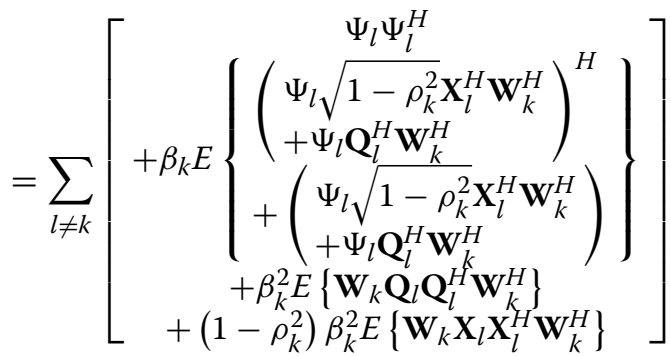

$$
\begin{aligned}
& \equiv \sum_{l \neq k}\left(\Psi_{l} \Psi_{l}^{H}+\mathbf{C}_{l, 1}+\mathbf{C}_{l, 2}+\mathbf{C}_{l, 3}\right)
\end{aligned}
$$

where $\mathbf{Q}_{l} \equiv \rho_{k} \hat{\mathbf{H}}_{k} \overline{\mathbf{B}}_{l}-\rho_{k} \hat{\mathbf{H}}_{k} \mathbf{B}_{k} \Psi_{l}$ and $\Psi_{l}$ and $\mathbf{Q}_{l}$ are known to the M-BS. Since $\mathbf{X}_{l}$ and $\mathbf{Z}_{k}$ are independent of each other, $\mathbf{C}_{l, 3}$ can be represented as

$$
\begin{aligned}
\mathbf{C}_{l, 3} & =\left(1-\rho_{k}^{2}\right) \beta_{k}^{2} E\left\{\mathbf{W}_{k} \Gamma_{l} \mathbf{W}_{k}^{H}\right\} \\
& =\left(1-\rho_{k}^{2}\right) \beta_{k}^{2} \operatorname{tr}\left(\mathbf{Y}_{l}\right) E\left\{\mathbf{W}_{k} \mathbf{W}_{k}^{H}\right\}
\end{aligned}
$$

where

$$
\begin{aligned}
\Gamma_{l} & =E\left\{\mathbf{X}_{l} \mathbf{X}_{l}^{H}\right\} \\
& =\operatorname{tr}\left(\mathbf{Y}_{l}\right) \mathbf{I} .
\end{aligned}
$$


As described in the previous section, we cannot represent the expectation of $\mathbf{W}_{k} \mathbf{W}_{k}^{H}$ in a closed form. Instead, we consider the SINR when $\rho_{k} \rightarrow 0$ and $\rho_{k} \rightarrow 1$ where the diagonal elements of $\mathbf{W}_{k} \mathbf{W}_{k}^{H}$ can be properly approximated. When $\rho_{k} \rightarrow 0, \mathbf{C}_{l, 1}$ and $\mathbf{C}_{l, 2}$ can be ignored, and the SINR of the $i$ th stream of S-BS $k$ can be represented as

$$
\gamma_{k, i}=\frac{\left[\mathbf{P}_{k}^{2}\right]_{i i}}{\left[\sum_{l \neq k}\left(\Psi_{l} \Psi_{l}^{H}+\mathbf{C}_{l, 3}\right)+\sigma_{n}^{2} E\left(\mathbf{W}_{k} \mathbf{W}_{k}^{H}\right)\right]_{i i}}
$$

The $i$ th diagonal element of $\mathbf{W}_{k} \mathbf{W}_{k}^{H}$ can be represented as [25]

$$
\begin{aligned}
{\left[E\left\{\mathbf{W}_{k} \mathbf{W}_{k}^{H}\right\}\right]_{i i} } & =\left[E\left\{\left(\beta_{k}^{2} \mathbf{B}_{k}^{H} \mathbf{H}_{k}^{H} \mathbf{H}_{k} \mathbf{B}_{k}\right)^{-1}\right\}\right]_{i i} \\
& =\beta_{k}^{-2} E\left\{\frac{\left|\left(\mathbf{G}_{k}^{(i-)}\right)^{H} \mathbf{G}_{k}^{(i-)}\right|}{\left|\mathbf{G}_{k}^{H} \mathbf{G}_{k}\right|}\right\}
\end{aligned}
$$

where $\mathbf{G}_{k} \equiv \mathbf{H}_{k} \mathbf{B}_{k}, \mathbf{G}_{k}^{(i-)}$ denotes $\mathbf{G}_{k}$ without the $i$ th column and $|\cdot|$ denotes the determinant of a matrix. It can be shown that when $\rho_{k} \rightarrow 0$, the column vectors of $\mathbf{Z}_{k} \mathbf{B}_{k}$ are independent of each other since the beam weight of the proposed scheme converges to eigenvectors of $\mathbf{R}_{k}$. Thus, (31) can be rewritten as

$$
\left[E\left\{\mathbf{W}_{k} \mathbf{W}_{k}^{H}\right\}\right]_{i i} \approx \beta_{k}^{-2} \frac{E\left\{\left|\left(\mathbf{G}_{k}^{(i-)}\right)^{H} \mathbf{G}_{k}^{(i-)}\right|\right\}}{E\left\{\left|\mathbf{G}_{k}^{H} \mathbf{G}_{k}\right|\right\}}
$$

The average determinant of the non-central Wishart matrix, $\mathbf{G}_{k}^{H} \mathbf{G}_{k}$, can be written as [26]

$$
\begin{aligned}
E\left\{\left|\mathbf{G}_{k}^{H} \mathbf{G}_{k}\right|\right\} & =\left|\left(1-\rho_{k}^{2}\right) \overline{\mathbf{R}}_{k}\right| 2^{s_{k}} \frac{\Gamma_{s_{k}}\left(\frac{N_{R}}{2}+1\right)}{\Gamma_{s_{k}}\left(\frac{N_{R}}{2}\right)} \\
& \cdot{ }_{1} F_{1}\left(-1 ; \frac{N_{R}}{2} ;-\frac{0.5 \rho_{k}^{2}}{1-\rho_{k}^{2}} \Omega_{k}\right) \\
& \equiv \zeta_{k}
\end{aligned}
$$

where $\overline{\mathbf{R}}_{k}=\mathbf{B}_{k}^{H} \mathbf{R}_{k} \mathbf{B}_{k}, \quad \Omega_{k}=\overline{\mathbf{R}}_{k}^{-1} \mathbf{B}_{k}^{H} \hat{\mathbf{H}}_{k}^{H} \hat{\mathbf{H}}_{k} \mathbf{B}_{k}$, $\Gamma_{s_{k}}(\cdot)$ denotes the multivariate gamma function and ${ }_{1} F_{1}(\cdot)$ denotes the hypergeometric function of matrix arguments. Similarly, it can be shown that

$$
\begin{aligned}
E\left\{\left|\left(\mathbf{G}_{k}^{(i-)}\right)^{H} \mathbf{G}_{k}^{(i-)}\right|\right\} & =\left|\left(1-\rho_{k}^{2}\right) \overline{\mathbf{R}}_{k}^{(i-)}\right| 2^{s_{k}-1} \\
& \cdot \frac{\Gamma_{s_{k}-1}\left(\frac{N_{R}}{2}+1\right)}{\Gamma_{s_{k}-1}\left(\frac{N_{R}}{2}\right)} \\
& \cdot{ }_{1} F_{1}\left(-1 ; \frac{N_{R}}{2} ;-\frac{0.5 \rho_{k}^{2}}{1-\rho_{k}^{2}} \Omega_{k}^{(i-)}\right) \\
& \equiv \zeta_{k}^{(i-)}
\end{aligned}
$$

where $\overline{\mathbf{R}}_{k}^{(i-)}=\left(\mathbf{B}_{k}^{(i-)}\right)^{H} \mathbf{R}_{k} \mathbf{B}_{k}^{(i-)}$ and $\Omega_{k}^{(i-)}=$ $\overline{\mathbf{R}}_{k}^{-1}\left(\mathbf{B}_{k}^{(i-1)}\right)^{H} \hat{\mathbf{H}}_{k}^{H} \hat{\mathbf{H}}_{k} \mathbf{B}_{k}^{(i-)}$. When $\rho_{k} \rightarrow 0$, the SINR can be approximated as

$$
\hat{\gamma}_{k, i}^{(0)}=\frac{\left[\mathbf{P}_{k}^{2}\right]_{i i}}{\left[\sum_{l \neq k} \Psi_{l} \Psi_{l}^{H}\right]_{i i}+\left\{\left(\rho_{k}^{2}\right) \sum_{l \neq k} \operatorname{tr}\left(\mathbf{Y}_{l}\right)+\beta_{k}^{-2} \sigma_{n}^{2}\right\} \frac{\zeta_{k}^{(i-)}}{\zeta_{k}}} .
$$

On the other hand, when $\rho_{k} \rightarrow 1, \mathbf{C}_{l, 1}$ and $\mathbf{C}_{l, 2}$ can be approximated as, respectively,

$$
\begin{aligned}
\mathbf{C}_{l, 1} & \approx\left(\Psi_{l} \mathbf{Q}_{l}^{H}\left\{\rho_{k} \mathbf{B}_{k}^{H} \hat{\mathbf{H}}_{k}^{H}\right\}^{\dagger}\right) \\
& +\left(\Psi_{l} \mathbf{Q}_{l}^{H}\left\{\rho_{k} \mathbf{B}_{k}^{H} \hat{\mathbf{H}}_{k}^{H}\right\}^{\dagger}\right)^{H} \\
& \equiv \hat{\mathbf{C}}_{l, 1}, \\
\mathbf{C}_{l, 2} & \approx\left(\rho_{k} \hat{\mathbf{H}}_{k} \mathbf{B}_{k}\right)^{\dagger} \mathbf{Q}_{l} \mathbf{Q}_{l}^{H}\left(\rho_{k} \mathbf{B}_{k}^{H} \hat{\mathbf{H}}_{k}^{H}\right)^{\dagger} \\
& \equiv \hat{\mathbf{C}}_{l, 2} .
\end{aligned}
$$

It can be shown that $\mathrm{C}_{l, 3}$ is the same as that when $\rho_{k} \rightarrow 0$. When $\rho_{k} \rightarrow 1$, the SINR, denoted by $\hat{\gamma}_{k, i}^{(1)}$, can approximately be represented as

$$
\hat{\gamma}_{k, i}^{(1)}=\frac{\left[\mathbf{P}_{k}^{2}\right]_{i i}}{\left[\sum_{l \neq k} \mathbf{A}_{l}\right]_{i i}+\left\{\left(1-\rho_{k}^{2}\right) \sum_{l \neq k} \operatorname{tr}\left(\mathbf{Y}_{l}\right)+\beta_{k}^{-2} \sigma_{n}^{2}\right\} \frac{\zeta_{k}^{(i-)}}{\zeta_{k}}}
$$

where $\mathbf{A}_{l} \equiv \Psi_{l} \Psi_{l}^{H}+\hat{\mathbf{C}}_{l, 1}+\hat{\mathbf{C}}_{l, 2}$.

When $0<\rho_{k}<1$, the numerator and the denominator of (31) are not independent of each other, making it difficult to get an approximated representation. However, simulation results show that $\hat{\gamma}_{k, i}^{(1)}$ and $\hat{\gamma}_{k, i}^{(0)}$ are valid for $0<\rho_{k}<1$. When $0<\rho_{k}<1$, the SINR of the $i$ th stream of S-BS $k$ can approximately be represented as

$$
\hat{\gamma}_{k, i}= \begin{cases}\hat{\gamma}_{k, i}^{(1)}, & \rho_{k} \geq \sqrt{0.5} \\ \hat{\gamma}_{k, i}^{(0)}, & \rho_{k}<\sqrt{0.5}\end{cases}
$$


where the threshold value $\sqrt{0.5}$ is determined from $\rho_{k}=$ $\sqrt{1-\rho_{k}^{2}}$ in (2).

Finally, we allocate the transmit power by means of iterative water-filling [27] with the use of average SINR by (39). The procedure is summarized in Table 1 , where $p_{k, i}$ is the $i$ th diagonal element of $\mathbf{P}_{k}$ and $N_{p}$ is the number of iterations for the water-filling.

\subsection{Complexity of the proposed scheme}

For the generation of beam weight by (22), the eigendecomposition in (11) and (21) may require a computational complexity of $O\left(N_{T}^{3}\right)$, which is similar to that of conventional ST-CSI-based SVD transmission scheme [10]. The only difference comes from the linear combination of ST-CSI and LT-CSI in (11) and (21), which may require a computational complexity of $O\left(N_{T}^{2}\right)$.

For the power allocation, the M-BS calculates $\hat{\gamma}_{k, i}^{(1)}$ (or $\left.\hat{\gamma}_{k, i}^{(0)}\right)$ and the maximum number of beams for each SMC is $s_{k}=N_{R}$. The SINR can be initialized with a computational complexity of $O\left(N_{T} N_{R}^{2}\right)$. It may require a computational complexity of $5 N_{T}^{2} N_{R}+10 N_{T} N_{R}^{2}+21 N_{R}^{3}+$ $9 N_{R}^{2}+N_{R}+O\left(P_{N_{h} N_{R}}^{2} N_{R}\right)$ for the estimation of $\hat{\gamma}_{k, i}^{(1)}$, where $O\left(P_{N_{h} N_{R}}^{2} N_{R}\right)$ is the computational complexity for the hypergeometric function in (33) and (34), $P_{N_{h} N_{R}}^{2} \sim$ $O\left(\exp \left(2 \pi \sqrt{2 N_{h} / 3}\right)\right)$ and $N_{h}$ is the number of iterations [28]. The power matrices are only required to be updated for each iteration, not the beam weight and the hypergeometric function. The procedure may require a computational complexity of $O\left(N_{R}^{2}\right)$ for each iteration, which is relatively small compared to that of the generation of beam weight.

Since $N_{T} \gg N_{R}$ in the system model, the proposed scheme may require an additional computational complexity of $O\left(N_{T}^{2}\right)$. This means that the computational complexity of the proposed scheme mainly depends upon the generation of beam weight requiring a computational complexity of $O\left(N_{T}^{3}\right)$, which is almost the same as that of the conventional scheme. However, the computational complexity for the hypergeometric function increases sub-exponentially proportional to $N_{h}$. The hypergeometric function is only used in the power allocation, not in the beam weight calculation. It was shown that the performance degradation of the power allocation is small when the interference terms in SINR is approximated to zero [10]. Although it did not consider temporal channel variation [10], it can be applied to estimate the SINR without using the hypergeometric function terms for the power allocation.

\section{Simulation results and discussions}

We verify the performance of the proposed scheme by computer simulation. The main simulation parameters are summarized in Table 2. We estimate the SINR by (39), where we approximately calculate the hypergeometric function by means of finite-series approximation [28]. For fair comparison, we apply the same water-filling algorithm to the conventional schemes in the simulation.

Figure 2 depicts the average spectral efficiency of the backhaul link according to temporal channel correlation $\rho_{k}$ when the number of S-BS antennas is 4 or 8 , where "AJD" refers to the proposed scheme when $\hat{\mathbf{V}}_{B}^{*}$ is determined by the AJD of (22), "perfect CSI" refers to the proposed scheme with $\mathbf{V}_{B}^{*}$ determined using perfect $\mathbf{Z}_{k}$, and "analysis" refers to the analysis by (39). It can be seen that the proposed and the AJD scheme may suffer from performance degradation due to unknown $\mathbf{Z}_{k}$. However, the proposed scheme with approximation by (22) provides performance similar to AJD, significantly reducing the computational complexity. The AJD requires large computational complexity to generate samples of the channel matrix and determines $\hat{\mathbf{V}}_{B}^{*}$ by an iterative algorithm [24], but the proposed scheme simply determines $\hat{\mathbf{V}}_{B}^{*}$ by an identity matrix. It can also be seen that the analysis agrees quite well with the simulation results.

Table 2 Main simulation parameters

\begin{tabular}{ll}
\hline Parameters & Values \\
\hline CCM & 3 -D ray-based model [29] \\
Carrier frequency & $2.3(\mathrm{GHz})$ \\
Cell radius & $200(\mathrm{~m})$ \\
Number of SMCs $(K)$ & 4 \\
SMC location & Uniformly random \\
Number of BS antennas $\left(N_{T}\right)$ & $64(2$-dimensional, $8 \times 8)$ \\
$\begin{array}{l}\text { Number of iterations for } \\
\text { water-filling }\left(N_{p}\right)\end{array}$ & 2 \\
$\begin{array}{l}\text { Number of iterations in the } \\
\text { hypergeometric function }\left(N_{h}\right)\end{array}$ & 20 \\
\hline
\end{tabular}

Table 1 Power allocation procedure

Input: $\hat{\mathbf{H}}_{k}, \mathbf{R}_{k}, \rho_{k}$
Initialization:
1: Obtain $\mathbf{B}_{k}$ for $\forall k$ by (22)
2: Initialize SINR as $\hat{\gamma}_{k, i}=\frac{\tilde{\mathbf{D}}_{k}^{2}}{\sigma_{n}^{2} \operatorname{tr}\left(\mathbf{B}_{k}^{H} \mathbf{B}_{k}\right)}$
3: Initialize $p_{k, i}$ by means of water-filling
Repeat $N_{p}$ times:
4: Update $\hat{\gamma}_{k, i}$ by (39)
5: Update $p_{k, i}$ by means of water-filling
6: Normalize the power by $p_{k, i} \leftarrow p_{k, i} \sqrt{\frac{\operatorname{tr}\left(\mathbf{P}_{k}^{2}\right)}{\operatorname{tr}\left(\overline{\mathbf{B}}_{k}^{+} \mathbf{B}_{k}\right)}}$
Output: $\mathbf{P}_{k}$



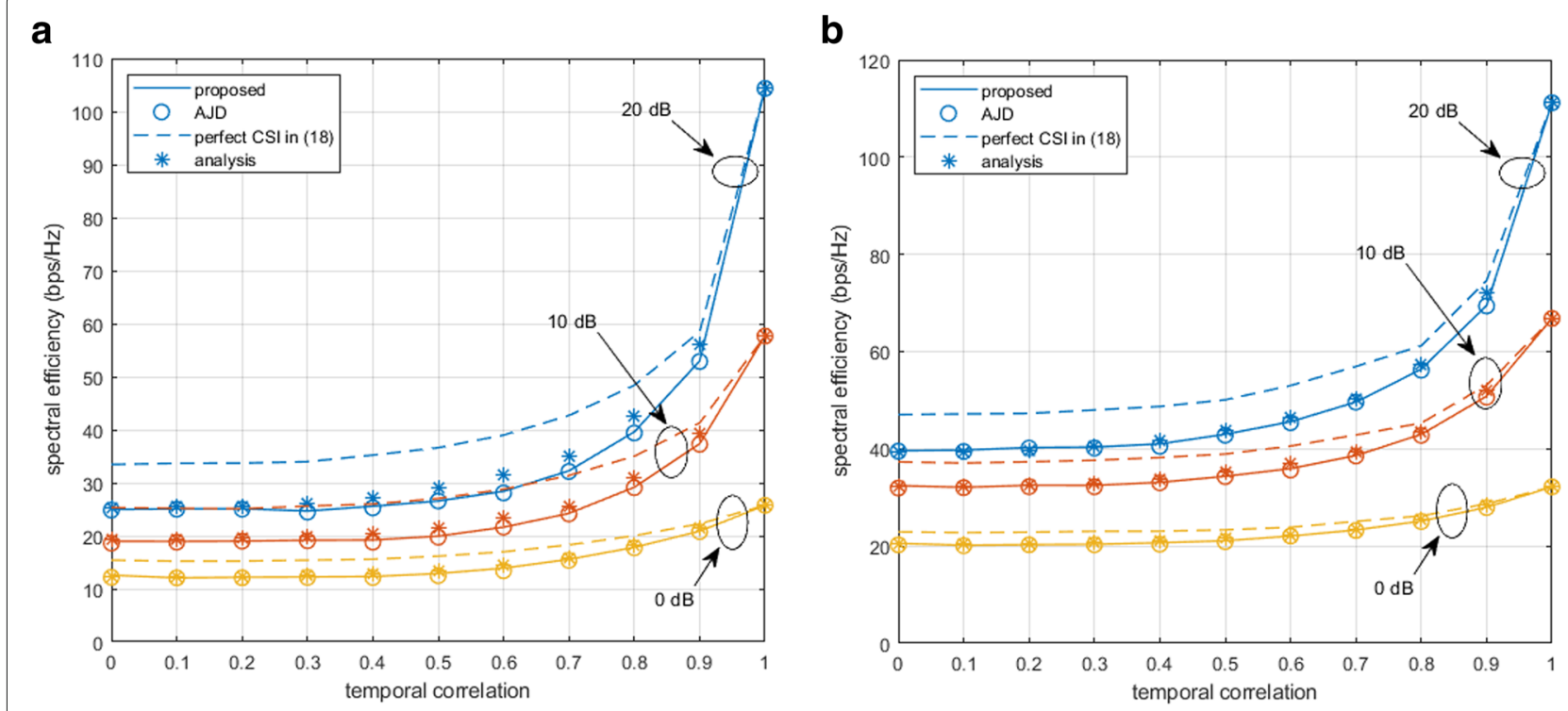

Fig. 2 Spectral efficiency according to channel variation. a When $N_{R}=4$. b When $N_{R}=8$

We compare the spectral efficiency of the proposed scheme with that of two-stage beamforming [10], statistical beamforming [11] and hybrid beamforming scheme [13], referred to as "ST-CSI-based," "LT-CSI-based," and "hybrid," respectively. The two-stage beamforming only exploits ST-CSI, where the outer beam weight is determined by a set of eigenvectors of interference channel matrix and the inner beam weight as a set of eigenvectors of the effective channel equal to the product of the original channel and the outer beam weight.
This scheme is identical to the proposed scheme when $\rho_{k}=1$. The statistical beamforming only exploits LTCSI, where the beam weight is determined by a set of eigenvectors of CCM corresponding to the largest eigenvalue. Since the conventional scheme only considers users equipped with a single antenna, it determines the beam weight in a two-stage manner, which is the same as the proposed scheme when $\rho_{k}=0$. The hybrid beamforming exploits ST- and LT-CSI to determine the inner and the outer beam weights, which are equal to

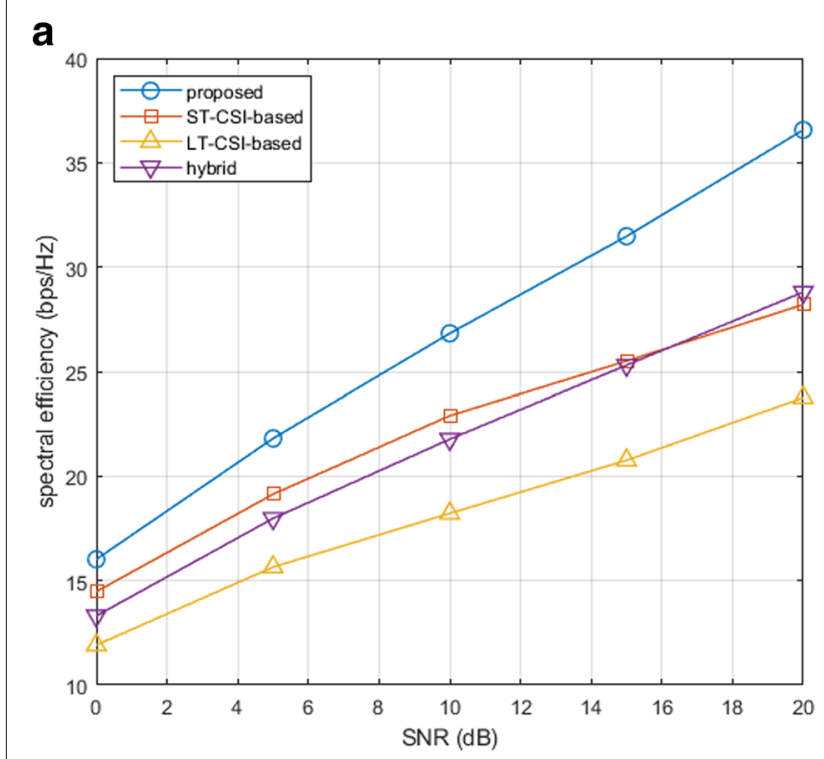

b

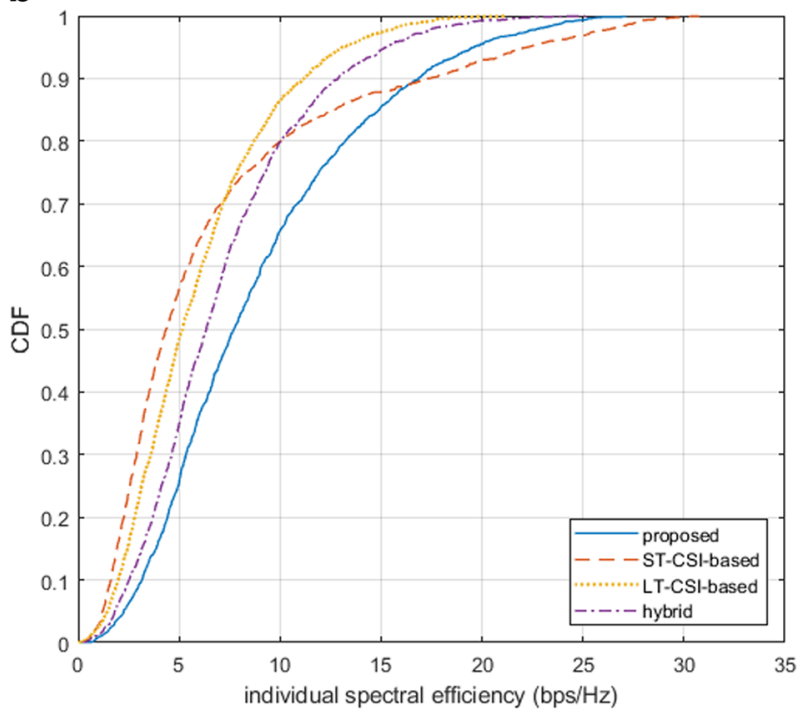

Fig. 3 Spectral efficiency in random velocity. a Spectral efficiency. b Empirical CDF of spectral efficiency 

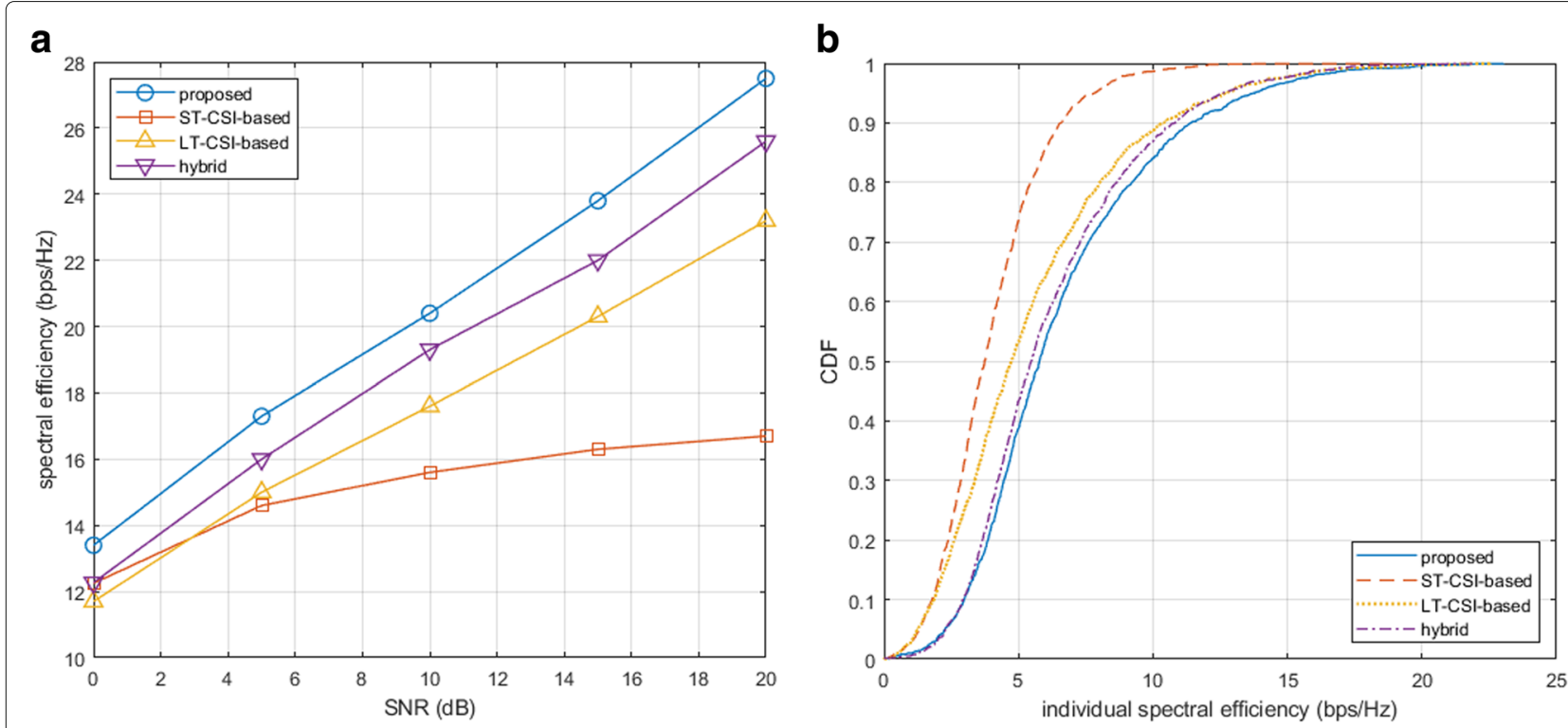

Fig. 4 Spectral efficiency in fixed velocity. a Spectral efficiency. b Empirical CDF of spectral efficiency

$\mathbf{B}_{k, 2}$ with $\rho_{k}=1$ and $\mathbf{B}_{k, 1}$ with $\rho_{k}=0$ in (22), respectively.

Figure 3 depicts the performance when each SMC is moving at a speed of uniformly distributed in a range of $(0$, $60) \mathrm{km} / \mathrm{h}$, and Fig. 4 depicts the performance when each $\mathrm{SMC}$ is moving at a fixed velocity of $30 \mathrm{~km} / \mathrm{h}$. The cumulative distribution function (CDF) of spectral efficiency of an SMC is empirically depicted when the average SNR is $20 \mathrm{~dB}$. The delay between the channel estimation and the signal transmission is set to $5 \mathrm{~ms}$ [30] and $N_{R}=4$. It can be seen from Fig. 3 that the proposed scheme outperforms the other schemes in a wide range of SNR. The LT-CSI-based scheme shows the worst performance since achievable beamforming gain in low mobility dominates the average transmission performance as seen in Fig. 2. By the same reason, the ST-CSI-based scheme shows better performance even though the SMCs suffer from the channel aging effect in the presence of high SMC mobility. It can be seen from Fig. 4 that the hybrid beamforming outperforms ST-CSI- and LT-CSI-based schemes. The STCSI-based schemes provide the worst performance mainly due to the channel mismatch problem, while the LT-CSIbased schemes provide poor performance since they do not exploit beamforming gain from ST-CSI. The proposed and the hybrid schemes yield empirical CDF similar to each other. However, the proposed scheme can provide higher spectral efficiency than the hybrid scheme, by adjusting the combine weight of ST-and LT-CSI according to the SMC mobility.

\section{Conclusions}

We have considered signal transmission in the wireless backhaul links for SMCs deployed in a cellular system.
We have determined the beam weight to maximize the average SLNR by exploiting ST- and LT-CSI. By transmitting data in a weighted eigen-direction of ST- and LT-CSI, the proposed scheme can provide transmission performance robust to the variation of SMC mobility. It adjusts the transmit power based on the estimated SINR. The simulation results show that the proposed scheme is quite efficient in the backhaul link.

\section{Appendix}

Let $\mathbf{U} \in \mathbb{C}^{N \times N}$ be a unitary matrix, $\mathbf{D} \in \mathbb{C}^{N \times N}$ be a diagonal matrix with distinct positive elements, $u_{i j}$ be an element of $\mathbf{U}$ corresponding to the $i$ th row and the $j$ th column, and $d_{i}$ be the $i$ th diagonal element of $\mathbf{D}$. Assume that the elements in D are sorted in an ascending order. It can be shown that

$$
\left[\mathbf{U D U}^{H}\right]_{i i}^{-1}=\frac{1}{\sum_{j=1}^{N} d_{i}\left|u_{i j}\right|^{2}} \equiv \frac{1}{\eta_{i}}
$$

where $u_{i j}$ is constrained by

$$
\left\{\begin{array}{l}
0 \leq\left|u_{i j}\right|^{2} \leq 1 \\
\sum_{i=1}^{N}\left|u_{i j}\right|^{2}=1 \text { and } \sum_{j=1}^{N}\left|u_{i j}\right|^{2}=1
\end{array} .\right.
$$

Let $\hat{\mathbf{U}}=\mathbf{I}_{N}$ with corresponding element $\hat{u}_{i j}$. It can be shown that

$$
\left\{\begin{array}{l}
\eta_{1}-\hat{\eta}_{1} \geq 0 \\
\sum_{i=1}^{N}\left(\eta_{i}-\hat{\eta}_{i}\right) \geq 0
\end{array}\right.
$$


where $\hat{\eta}_{i}=\sum_{j=1}^{N} d_{i}\left|\hat{u}_{i j}\right|^{2}$. By the convexity of (40), it can be shown that

$$
\sum_{i=1}^{N} \frac{1}{\eta_{i}} \leq \sum_{i=1}^{N} \frac{1}{\hat{\eta}_{i}}=\sum_{i=1}^{N} \frac{1}{d_{i}} .
$$

When $\mathbf{D}$ is not a diagonal matrix, it can be diagonalized by representing $\mathbf{U}=\overline{\mathbf{U}}_{D}^{H}$, where $\mathbf{U}_{D}$ is an eigenvector matrix of $\mathbf{D}$ and $\overline{\mathbf{U}}$ is a unitary matrix. Then, it can be shown that the equality in (43) holds when $\mathbf{U}=\mathbf{U}_{D}^{H}$.

\section{Abbreviations}

BS: Base station; CCM: Channel correlation matrix; CDF: Cumulative distribution function; CSI: Channel state information; DM-RS: Demodulation reference signal; LT-CSI: Long-term CSI; MIMO: Multi-input multi-output; NLOS: Non-line-of-sight; QoS: Quality of service; S-BS: Small moving cell BS; SINR: Signal-to-interference-plus-noise ratio; SLNR: Signal-to-leakage-plus-noise ratio; SMC: Small moving cell; SNR: Signal-to-noise ratio; ST-CSI: Short-term CSI; SVD: Singular value decomposition; VPL: Vehicular penetration loss; ZF: Zero-forcing

\section{Acknowledgements}

This work was supported by the National Research Foundation of Korea(NRF) grant funded by the Korea government(MSIT) (No. 2019R1F1A1063171).

\section{Authors' contributions}

HK conceived and designed the study. HK, YB, and GJ performed experiments and analyzed the experimental results. YL directed the academic research. HK wrote the paper, and $Y L$ revised the manuscript. All authors read and approved the manuscript.

\section{Availability of data and materials}

The first author has the data and source codes.

\section{Competing interests}

The authors declare that they have no competing interests.

\section{Author details}

${ }^{1}$ School of Electrical Engineering and INMC, Seoul National University, Gwanak-ro, Seoul, Republic of Korea. ${ }^{2}$ School of Electrical Engineering and INMC, Seoul National University, Gwanak-ro, Seoul, Republic of Korea.

Received: 11 April 2019 Accepted: 10 September 2019

Published online: 24 October 2019

\section{References}

1. D. Gesbert, et al., Shifting the MIMO paradigm. IEEE Signal Process. Mag. 24(5), 36-46 (2007)

2. T. Shim, et al., Traffic convexity aware cellular networks: a vehicular heavy user perspective. IEEE Wirel. Commun. 23(1), 88-94 (2016)

3. Y. Sui, et al., Moving cells: a promising solution to boost performance for vehicular users. IEEE Intell. Transp. Syst. Mag. 51(6), 62-68 (2013)

4. A. Jaziri, R. Nasri, T. Chahed, in Proceedings of ICC: 22-27 May 2016. Offloading traffic hotspots using moving small cells, (Kuala Lumpur, 2016), pp. 1-6. https://doi.org/10.1109/icc.2016.7511566

5. F. Haider, et al., Spectral/energy efficiency tradeoff of cellular systems with mobile femtocell deployment. IEEE Trans. Veh. Tech. 65(5), 3389-3400 (2016)

6. S. Jaffry, S. F. Hasan, X. Gui, in Proceedings of GLOBECOM: 9-13 Dec. 2018. Shared spectrum for mobile-cell's backhaul and access link, (Abu Dhabi, 2018), pp. 1-6. https://doi.org/10.1109/glocom.2018.8647131

7. S. T. Shah, et al., Moving personal-cell network: characteristics and performance evaluation. China Commun. 15(12), 159-173 (2018)

8. P. Harris, et al., Performance characterization of a real-time massive MIMO system with los mobile channels. IEEE J. Sel. Areas Commun. 35(6), 1244-1253 (2017)
9. J. Erman, et al., To cache or not to cache: the $3 G$ case. IEEE Internet Comput. 15(2), 27-34 (2011)

10. V. Stankovic, M. Haardt, Generalized design of multi-user MIMO precoding matrices. IEEE Trans. Wirel. Commun. 7(3), 953-961 (2008)

11. C. Zhang, et al., in Proceedings of ICCC: $2-4$ Nov. 2015. Statistical beamforming for FDD massive MIMO downlink systems, (Shenzhen, 2015), pp. 1-6. https://doi.org/10.1109/iccchina.2015.7448643

12. X. Li, S. Jin, R. W. Heath, $3 d$ beamforming for large-scale FD-MIMO systems exploiting statistical channel state information. IEEE Trans. Veh. Tech. 65(11), 8992-9005 (2016)

13. J. Nam, et al., Joint spatial division and multiplexing: opportunistic beamforming, user grouping and simplified downlink scheduling. IEEE J. Sel. Topics Signal Process. 8(5), 876-890 (2014)

14. L. Fan, et al., A low-complexity 3D massive MIMO scheme jointly using statistical and instantenous CSIT. EURASIP J. Wirel. Commun. Netw. 1, 235 (2016)

15. Y. Jeon, et al., New beamforming designs for joint spatial division and multiplexing in large-scale MISO multi-user systems. IEEE Trans. Wirel. Commun. 16(5), 3029-3041 (2017)

16. M. Sternad, et al., in Proceedings of WCNC: 1-1 April 2012. Using "predictor antennas" for long-range prediction of fast fading for moving relays, (Paris, 2012), pp. 253-257. https://doi.org/10.1109/wcncw.2012.6215501

17. D. P-Huy, M. Sternad, T. Svensson, Making $5 \mathrm{G}$ adaptive antennas work for very fast moving vehicles. IEEE Intell. Transp. Syst. Mag. 7(2), 71-84 (2015)

18. T. Li, et al., Position-aided large-scale MIMO channel estimation for high-speed railway communication systems. IEEE Trans. Veh. Tech. 66(10), 8964-8978 (2017)

19. N. Jamaly, T. Svensson, A. Derneryd, Effects of coupling and overspeeding on performance of predictor antenna systems in wireless moving relays. IET Microw. Antennas Propag. 13(3), 367-372 (2019)

20. W. C. Jakes, Microwave Mobile Communications. (Wiley, New York, 1974)

21. M. Sadek, A. Tarighat, A. H. Sayed, A leakage-based precoding scheme for downlink multi-user mimo channels. IEEE Trans. Wirel. Commun. 6(5), 1711-1721 (2007)

22. Y. Jiang, M. K. Varanasi, J. Li, Performance analysis of ZF and MMSE equalizers for MIMO systems: an in-depth study of the high SNR regime. IEEE Trans. Inform. Theory. 57(4), 2008-2026 (2011)

23. R. D. Cook, On the mean and variance of the generalized inverse of a singular Wishart matrix. Electron. J. Statist. 5, 146-158 (2011)

24. M. Congedo, et al., Approximate joint diagonalization and geometric mean of symmetric positive definite matrices. PLoS ONE. 10(4), 1-25 (2015)

25. K. K. Wong, Z. Pan, Array gain and diversity order of multiuser MISO antenna systems. Int. J. Wirel. Inform. Netw. 15(2), 82-89 (2008)

26. R. J. Muirhead, Aspects of Multivariate Statistical Theory. (Wiley, New York, 1982)

27. W. Yu, G. Ginis, J. M. Cioffi, Distributed multiuser power control for digital subscriber lines. IEEE J. Sel. Areas Commun. 20(5), 1105-1115 (2002)

28. P. Koev, A. Edelman, The efficient evaluation of the hypergeometric function of a matrix argument. Math. Comput. 75, 833-846 (2006)

29. D. Ying, et al., in Proceedings of ICC: 10-14 Aug. 2014. Kronecker product correlation model and limited feedback codebook design in a 3D channel model, (Sydney, 2014), pp. 5865-5870. https://doi.org/10.1109/ icc.2014.6884258

30. L. Thiele, et al., in Proceedings of ASILOMAR: 6-9 Nov. 2011. Channel aging effects in CoMP transmission: gains from linear channel prediction, (Pacific Grove, 2011), pp. 1924-1928. https://doi.org/10.1109/acssc.2011.6190359

\section{Publisher's Note}

Springer Nature remains neutral with regard to jurisdictional claims in published maps and institutional affiliations. 